\title{
Comparative Study of Integer and Non-Integer Order Models of Synchronous Generator
}

\author{
Szymon Racewicz ${ }^{1, *(1)}$, Filip Kutt ${ }^{2}\left(\mathbb{D}\right.$, Michał Michna $^{2}\left(\mathbb{D}\right.$ and Łukasz Sienkiewicz ${ }^{2}$ \\ 1 Faculty of Mechatronics and Technical-Computer Sciences Education, University of Warmia and Mazury in \\ Olsztyn, 10-719 Olsztyn, Poland \\ 2 Faculty of Electrical and Control Engineering, Gdańsk University of Technology, 80-233 Gdańsk, Poland; \\ filip.kutt@pg.edu.pl (F.K.); michal.michna@pg.edu.pl (M.M.); lukasz.sienkiewicz@pg.edu.pl (Ł.S.) \\ * Correspondence: szymon.racewicz@uwm.edu.pl
}

Received: 10 July 2020; Accepted: 24 August 2020; Published: 26 August 2020

\begin{abstract}
This article presents a comparison between integer and non-integer order modelling of a synchronous generator, in the frequency domain as well as in the time domain. The classical integer order model was compared to one containing half-order systems. The half-order systems are represented in a Park $d-q$ axis equivalent circuit as impedances modelled by half-order transmittances. Using a direct method based on the approximation of the half-order derivatives by the Grünwald-Letnikov definition, a state-space equation system was solved. For both models, a computational program written in Matlab ${ }^{\circledR}$ software was used. For the purpose of time domain simulation, the machine models were connected to an electric load composed of an $R L$ circuit. To validate and compare both models, simulation results of a three-phase short-circuit and a no-load voltage recovery were compared with corresponding measurements performed on a solid salient-pole synchronous generator of $125 \mathrm{kVA}$.
\end{abstract}

Keywords: fractional calculus; Grünwald-Letnikov definition; non-integer order modelling; synchronous generator

\section{Introduction}

Non-integer order modelling is generally used to model devices where diffusion phenomena are present and influence their functionality. These can be, for example, diffusions of temperature, electric field or gases. One such phenomenon is also diffusion of the magnetic field in a solid ferromagnetic material. In most electrical machines, induced currents that are proportional to the frequency increase can no longer be neglected. Therefore, half-order modelling has been proposed to better describe the behaviour of electrical machines susceptible to the presence of induced currents in some of their conductive parts. Previous works have introduced such modelling in mechanical engineering for car suspension modelling [1], in electrochemical engineering for modelling of batteries [2], fuel cells [3], capacitors [4], supercapacitors [5] and ultracapacitors [6], as well as in electrical engineering for modelling of induction machines [7], synchronous machines [8,9] or transformers [10]. Classically, equivalent circuits of electrical device models are enhanced by adding ladder elements with constant parameters, usually $R, L$ or $C$ (resistance, inductance and capacitance, respectively) [11,12]. However, in theory, to better represent the diffusion effect, which is a distributed phenomenon described by partial differential equations, one has to integrate into equivalent circuits an infinite number of such ladders. Practically, this number is a chosen finite to meet the desired accuracy over a studied frequency range [13]. The main advantage of using non-integer order models is their reduced order in comparison to the classical integer order ones. This is due to the fact that non-integer order transmittances are much closer in mathematical relation to the physics of the studied phenomena than integer 
order ones. For electrical machine models represented by equivalent circuits with non-integer order systems, the structure and circuit parameters have been directly derived from Maxwell equations [14]. Nevertheless, this compactness feature is most pronounced in the frequency domain. For the time domain analysis, the non-integer order derivatives present in the model need to be approximated, often by integer order systems [15]. It is then interesting to investigate the differences between these two modelling approaches, especially for the time domain.

In this article, time domain simulations of a synchronous generator modelled by equivalent circuits are performed using a direct method, which consists of replacing derivatives by their numerical definitions. For the non-integer order derivatives, the Grünwald-Letnikov approximation is applied [16-18].

The paper is organized as follows. First, the equivalent circuits of the integer and non-integer order models of the synchronous generator are recalled. Then, for both models, a state-space equation system is constructed. After the identification process in the frequency domain, the Bode diagrams of the operational inductances, as well as the identified parameters of both models, are compared. Finally, both models are validated using data from short-circuit and power restoration tests performed on a $125 \mathrm{kVA}$ synchronous generator.

\section{Integer and Non-Integer Order Models of Synchronous Generator}

Classical equivalent circuits of the synchronous generator are presented in Figure 1. This simple model includes one $R L$ ladder element responsible for modelling the skin effect of squirrel cage damper bars during transient states. Nevertheless, it does not take into account the eddy currents developed in massive parts of the machine. Such a model is then appropriate for low frequency range modelling. To increase the modelled frequency range by taking into account the skin and eddy current effects, the virtual windings of the damper and massive parts modelled by RL elements should be added to the $d$ and $q$ axes. The number of such elements is selected arbitrarily to achieve the assumed accuracy. This kind of model is widely used and described in the literature [19-23].
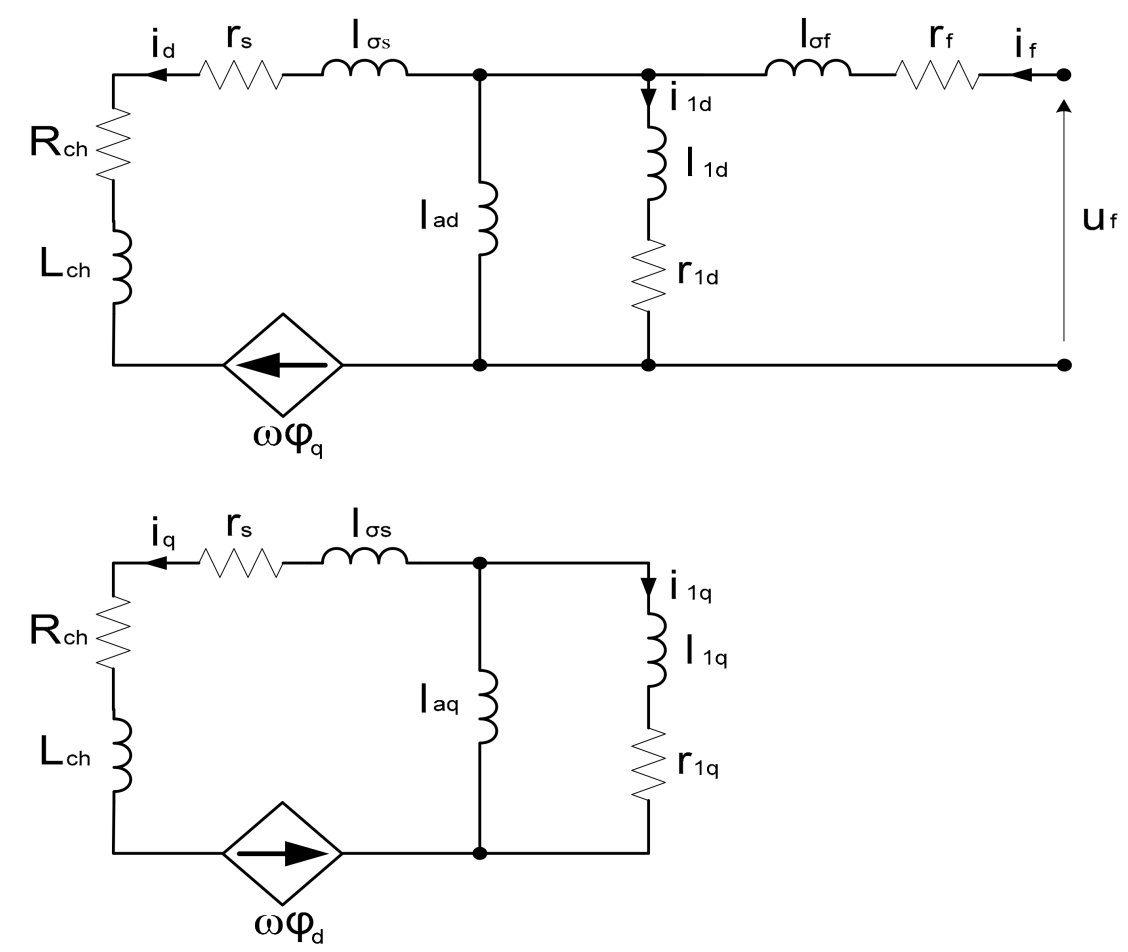

Figure 1. Classical integer order equivalent circuits of a synchronous generator connected to $R L$ electric load (axes $d$ and $q$ ). 
Another approach to increase the accuracy and frequency range of electric machine models is to replace the $R L$ elements present in the classical equivalent circuits with impedances described by half-order transmittances [24,25]. Figure 2 illustrates the non-integer order $d-q$ equivalent circuits of a synchronous generator with half-order impedances integrated into the model.
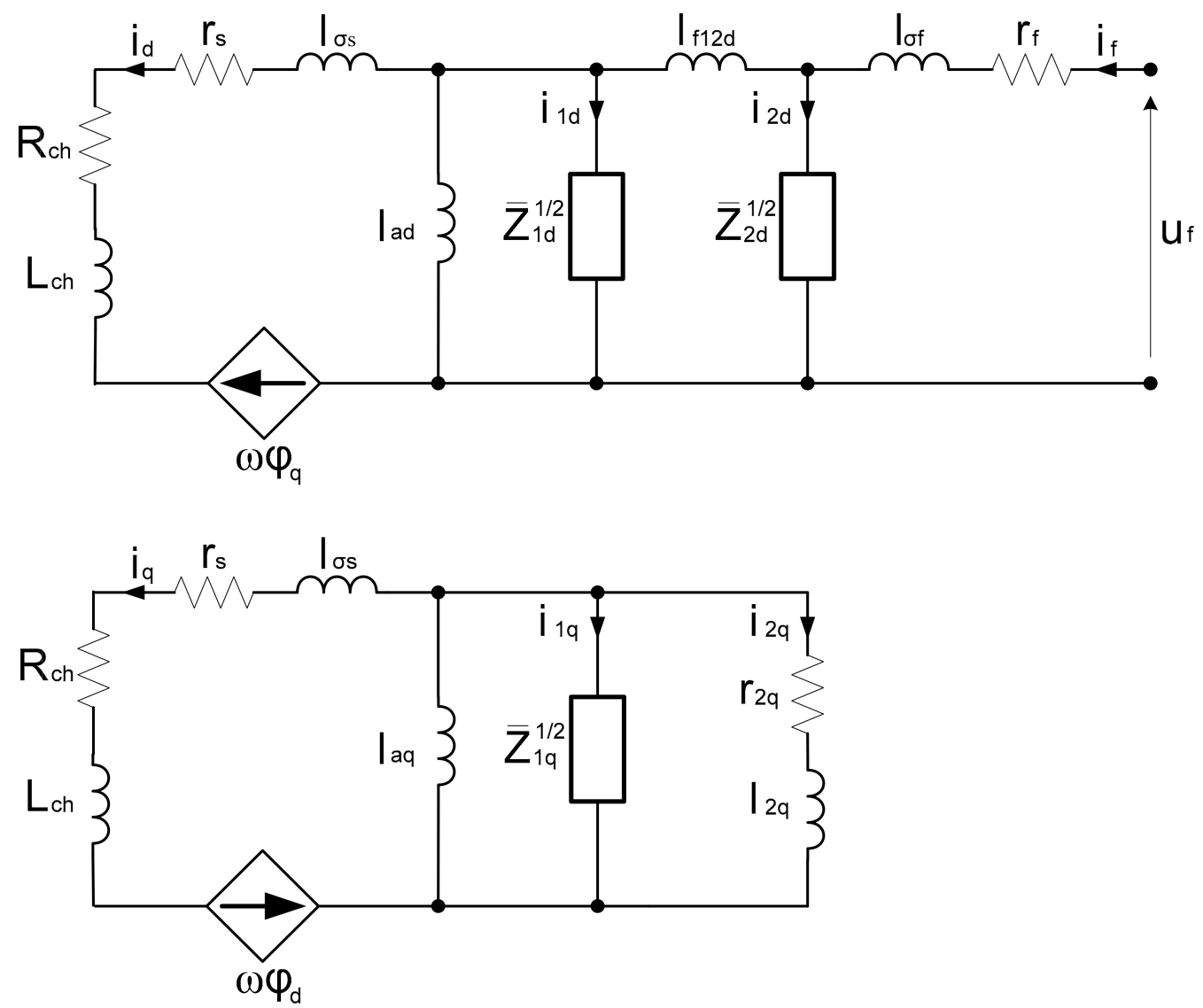

Figure 2. Non-integer order equivalent circuits of a synchronous generator connected to $R L$ electric load (axes $d$ and $q$ ).

The impedances described by half-order systems and those used in $d$ - $q$ equivalent circuits (Figure 2) are different. When modelling massive parts of the solid steel rotor, one should use the inductive impedance described by Equation (1). Meanwhile, for the description of squirrel cage damper bars, the resistive type of impedance is proposed by Equation (2) [26]. For the aim of identification, this model is simplified, i.e., damper bars' representation on the $q$ axis is described by the classic resistance $r_{2 q}$ and the classic inductance $l_{2 q}$, which are constant with frequency [27].

$$
\begin{aligned}
& \bar{Z}_{1 d / 1 q}^{1 / 2}(s)=\frac{L_{1 d / 1 q^{\cdot s}}}{\sqrt{1+\frac{s}{\omega_{1 d / 1 q}}}} \\
& \bar{Z}_{2 d}^{1 / 2}(s)=R_{2 d} \cdot \sqrt{1+\frac{s}{\omega_{2 d}}}
\end{aligned}
$$

The non-integer order model from 
Figure 2 is derived as follows:

$$
\left\{\begin{array}{c}
R_{c h} \cdot i_{d}+s \cdot L_{c h} \cdot i_{d}=-r_{s} \cdot i_{d}-s \cdot l_{\sigma s} \cdot i_{d}-s \cdot l_{a d} \cdot\left(i_{d}-i_{f}+i_{1 d}+i_{2 d}\right)-\omega \varphi_{q} \\
u_{f}=i_{f} \cdot r_{f}+s \cdot l_{\sigma f} \cdot i_{f}+s \cdot l_{f 12 d} \cdot\left(i_{f}-i_{2 d}\right)-s \cdot l_{a d} \cdot\left(i_{d}-i_{f}+i_{1 d}+i_{2 d}\right) \\
0=\bar{Z}_{1 d}^{1 / 2} \cdot i_{1 d}+s \cdot l_{a d} \cdot\left(i_{d}-i_{f}+i_{1 d}+i_{2 d}\right) \\
0=\bar{Z}_{2 d}^{1 / 2} \cdot i_{2 d}+s \cdot l_{a d} \cdot\left(i_{d}-i_{f}+i_{1 d}+i_{2 d}\right)-s \cdot l_{f 12 d} \cdot\left(i_{f}-i_{2 d}\right) \\
R_{c h} \cdot i_{q}+s \cdot L_{c h} \cdot i_{q}=-r_{s} \cdot i_{q}-s \cdot l_{\sigma s} \cdot i_{q}-s \cdot l_{a q} \cdot\left(i_{q}+i_{1 q}+i_{2 q}\right)+\omega \varphi_{d} \\
0=s \cdot l_{a q} \cdot\left(i_{q}+i_{1 q}+i_{2 q}\right)+r_{2 q} \cdot i_{2 q}+s \cdot l_{2 q} \cdot i_{2 q} \\
0=\bar{Z}_{1 q}^{1 / 2} \cdot i_{1 q}+s \cdot l_{a q} \cdot\left(i_{q}+i_{1 q}+i_{2 q}\right)
\end{array}\right.
$$

with flux components equal to:

$$
\begin{gathered}
\varphi_{d}=l_{\sigma s} \cdot i_{d}+l_{a d} \cdot\left(i_{d}-i_{f}+i_{1 d}+i_{2 d}\right) \\
\varphi_{q}=l_{\sigma s} \cdot i_{q}+l_{a q} \cdot\left(i_{q}+i_{1 q}+i_{2 q}\right)
\end{gathered}
$$

The implicit half-order transmittances of the impedances presented in Equations (1) and (2) are difficult to analyse analytically. Indeed, after integration into equation system (3), one obtains differential equations comprising Laplace operators to the power of 2 and higher, which complicates considerably the variables' change process and the expression of the model in the form of state-space Equation (6).

$$
\bar{x}^{(1 / 2)}=A \cdot \bar{x}+B \cdot \bar{u}
$$

where $A$ is the state matrix, $B$ is the input matrix, $\bar{x}$ is the state vector and $\bar{u}$ is the input vector.

Therefore, the implicit half-order transmittances (1) and (2) are replaced by their explicit equivalents given below:

$$
\begin{gathered}
\bar{Z}_{1 d}^{1 / 2}(s)=\frac{L_{1 d} \cdot s}{1+\sqrt{\frac{s}{\omega_{1 d}}}} \\
\bar{Z}_{1 q}^{1 / 2}(s)=\frac{L_{1 q} \cdot s}{1+\sqrt{\frac{s}{\omega_{1 q}}}} \\
\bar{Z}_{2 d}^{1 / 2}(s)=R_{2 d} \cdot\left(1+\sqrt{\frac{s}{\omega_{2 d}}}\right)
\end{gathered}
$$

This mathematical change does not have any great impact on the model precision, which has been proved in [27] by juxtaposing the two frequency models (implicit and explicit) of the operational inductances $\left(L_{d}, L_{q}\right)$.

Finally, integrating Equations (3)-(5) and (7)-(9), one obtains a system of non-integer order differential equations describing the synchronous generator with $R L$ load.

\section{Parameter Identification}

The parameter identification process for both integer and non-integer order models can be carried out based on input from the Standstill Frequency Response test (SSFR) $[25,28]$ and the least-squares problem procedures. Identification of parameters in the frequency domain is particularly useful for non-integer order models as the square roots of the Laplace operators present in the differential equations are interpreted as $\sqrt{j \cdot 2 \pi f}$ and so they are calculated fast and without any numerical problems. Figure 3 presents identification results for the $125 \mathrm{kVA}$ synchronous generator. 

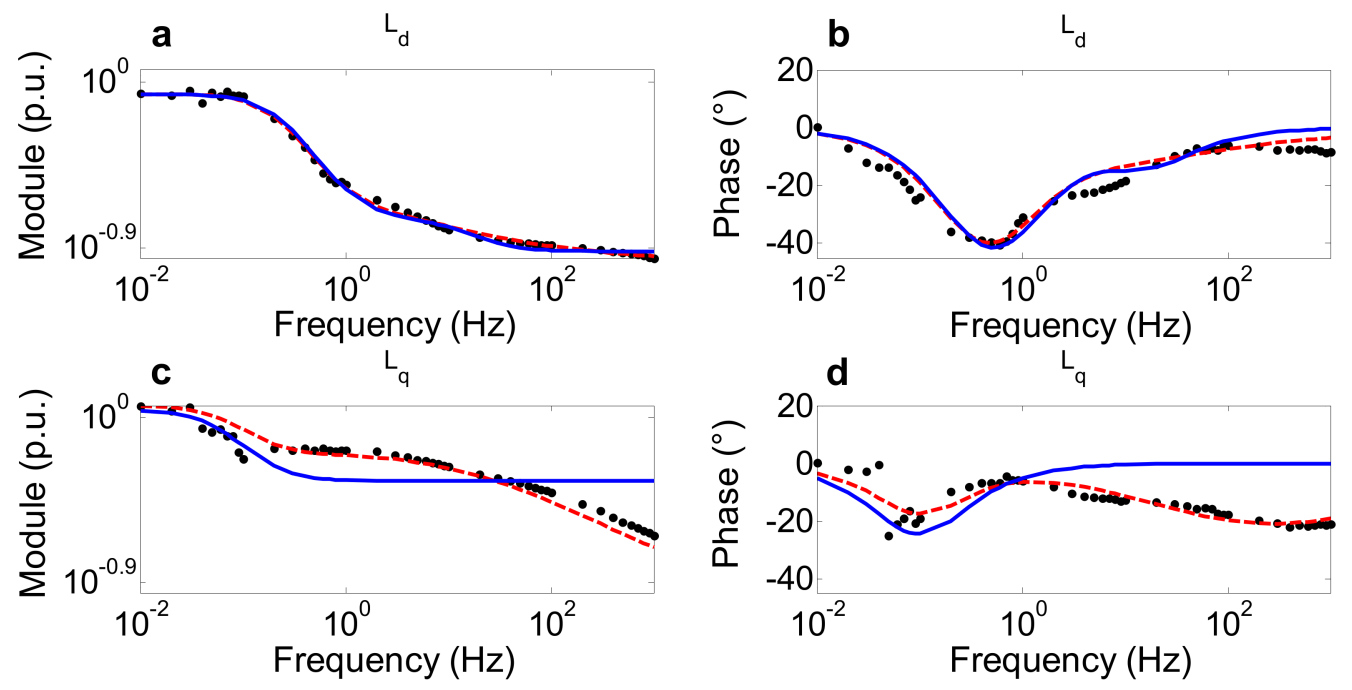

Figure 3. Bode diagrams of the $125 \mathrm{kVA}$ synchronous generator inductances $L_{d}$ and $L_{q}$ (black dots-measurements, blue solid line—integer order model, red dashed line—non-integer order model). (a) module of operational inductance $L_{d}$, (b) phase of operational inductance $L_{d}$, (c) module of operational inductance $L_{q}$, (d) phase of operational inductance $L_{q}$.

As shown in Figure 3, even with an approximation by the explicit half-order transmittances, the non-integer order model has smaller total error throughout the studied frequency range. Indeed, the classical integer order model with fewer parameters, especially on the $q$ axis, cannot be identified with equal accuracy. To improve the precision of the classical models, one has to expand the $d-q$ equivalent circuits with further ladder elements $(R L)$, which results in physical meaning loss of the circuit parameters that can in some cases take even negative values during the identification process.

One of the problems arising during global identification of all the parameters simultaneously using the full frequency range for the least-squares procedure is the numerical indistinguishability of model parameters. To overcome this problem, to some extent one has to suppose the probable ranges of parameters before initializing the identification process [19]. However, some of the parameters can have more impact on the model output than others, which results in different identification accuracy.

\section{Measurement Setup for Time Domain Validation}

To validate and compare integer and non-integer order models of the synchronous generator in the time domain, an Elmor GCh114a/4 3-phase, 4-pole, $125 \mathrm{kVA}(0.8 \mathrm{pf}), 400 \mathrm{~V}$ (Y connected) salient pole synchronous generator (the rated data is located in the Appendix A) was measured using a controlled short-circuit setup (Figure 4). The studied generator was driven by a dynamometer test bench with speed and torque control (Figure 5).

Measurements of the three-phase short-circuit from idle operation and no-load voltage recovery were performed. The voltage, current, speed and torque waveforms were acquired using LEM $^{\circledR}$ current transducers and a National Instruments USB data acquisition card controlled with PC software. 


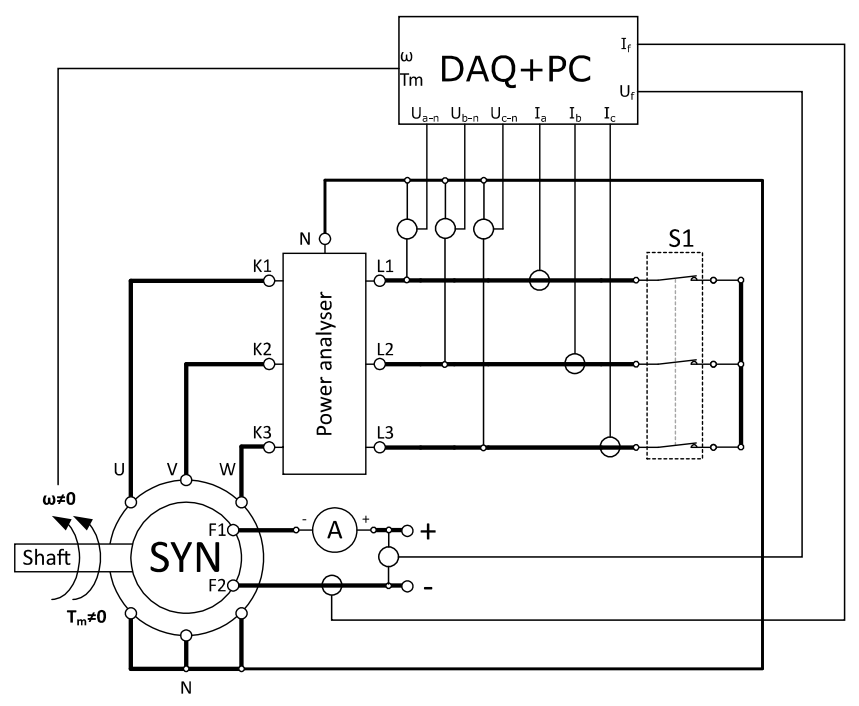

Figure 4. Diagram of the measurement setup for short-circuit and voltage recovery tests.

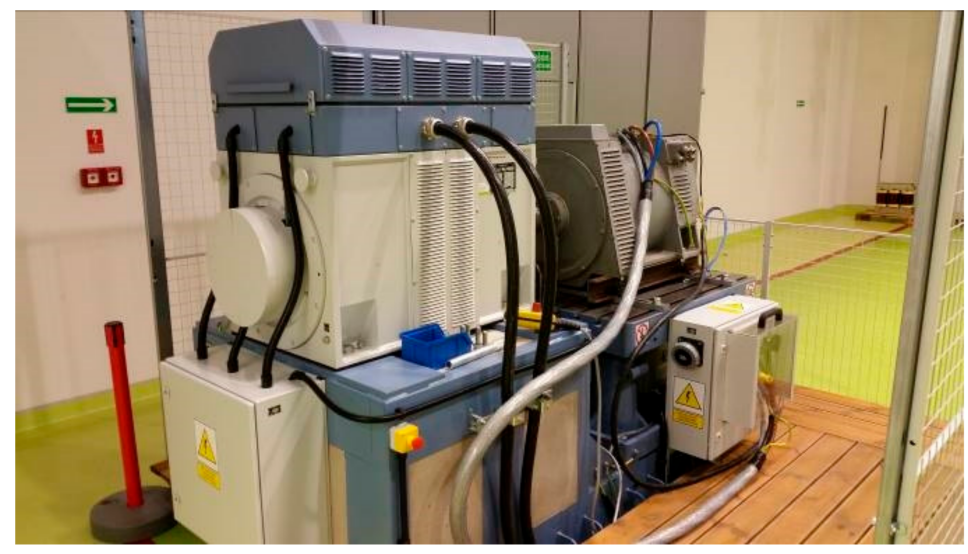

Figure 5. Measurement setup with the dynamometer (on the left) and the studied synchronous generator (on the right).

\section{Simulation and Measurement Results}

Solving the system of integer order differential equations is relatively simple. Indeed, integer order derivatives are well known and can be calculated using inbuilt procedures proposed by various simulation software or can be calculated numerically directly from the mathematical derivative definition. However, for models with non-integer order derivatives, one has to use original scripts with the mathematical definitions integrated inside. The most frequently used are the Grünwald-Letnikov, the Riemann-Liouville and the Caputo fractional derivative definitions [29]. Only during the last two decades have some numerical tools for the calculation of fractional operations appeared [30].

For this article, the original application coded in the Matlab ${ }^{\circledR}$ environment was utilized to compute the non-integer order differential equation system. The non-integer order derivatives were evaluated numerically by discretization using the Grünwald-Letnikov definition (10), which describes the $\alpha$-order derivative of a function $\mathrm{x}$ at a time $t_{m}=m \cdot h$.

$$
x^{(\alpha)}(m \cdot h)=\frac{1}{h^{\alpha}} \cdot\left\langle x(m \cdot h)+\sum_{k=1}^{m}\left\{(-1)^{k} \cdot\left(\begin{array}{l}
\alpha \\
k
\end{array}\right) \cdot x[(m-k) \cdot h]\right\}\right\rangle
$$

where

$$
\left(\begin{array}{c}
\alpha \\
k
\end{array}\right)=\frac{\alpha \cdot(\alpha-1) \cdot \ldots \cdot(\alpha-k+1)}{k !}
$$


The two prepared models of the $125 \mathrm{kVA}$ synchronous generator were used to simulate two different states of operation: the three-phase short-circuit from the no-load operation and the no-load voltage recovery. Sinusoidal currents $\left(i_{a}, i_{b}, i_{c}\right)$ in the natural axes $(a, b, c)$ were obtained by inverse Park transformation applied to the currents $i_{d}$ and $i_{q}$ [19].

Simulations of short-circuit and no-load voltage recovery were realized by changing the $R L$ load parameters $\left(R_{c h}, L_{c h}\right)$ from $1 \mathrm{e} 6 \Omega, 0 \mathrm{H}$, to $1 \mathrm{e}-3 \Omega, 0 \mathrm{H}$, and vice versa.

In the case of the non-integer order model, calculations of the non-integer order derivatives require a numerical history, i.e., some of the derivatives' values calculated backwards in time. Practically, the values calculated at the beginning of the simulation have an unacceptable error. On the other hand, too long a numerical history causes problems associated with so-called calculation tail [31]. Equation (12) describes an error caused by the limitation of the past derivatives' values taken into account for the non-integer order derivative calculation [16].

$$
\left(\begin{array}{l}
\alpha \\
k
\end{array}\right)=\frac{x_{M A X} \cdot K^{-\alpha}}{|\Gamma(1-\alpha)|}
$$

where $\alpha$ is the order of the derivative, $x_{\max }$ is the maximal value of the function $x(t), K$ is the number of values of the past derivative taken into account and $\Gamma$ is the gamma function. For the studied case, with the limitation of 2500 samples backwards, this error is equal to about $1.13 \%$ for all of the state variables' half-order derivatives.

To obtain an accurate approximation of the simulated states of operation, one has to simulate for some time before the transient state occurs. This provides the numerical history for the non-integer order derivative calculation and so reduces simulation error. Nevertheless, the time-consuming simulation process in the time domain is one of the biggest non-integer order model disadvantages.

Simulation results of the three-phase short-circuit test for the two studied models are presented and compared to the measurement data in Figures 6 and 7. The comparison emphasizes differences between the integer and non-integer order model behaviour during transient states of the synchronous generator operation, especially for the excitation current representation. This loss of precision is due to the sudden change in the numerical formulas' input, which cannot influence all of the numerical history of the non-integer order derivatives. However, after some time of calculation, one can see a satisfactory waveform compliance. Even if in the frequency domain the non-integer order model presents higher precision, its overall output error in the time domain is bigger than for the classical integer order one. Nevertheless, the stator and the excitation current waveforms' shape and values at steady state are rendered correctly.

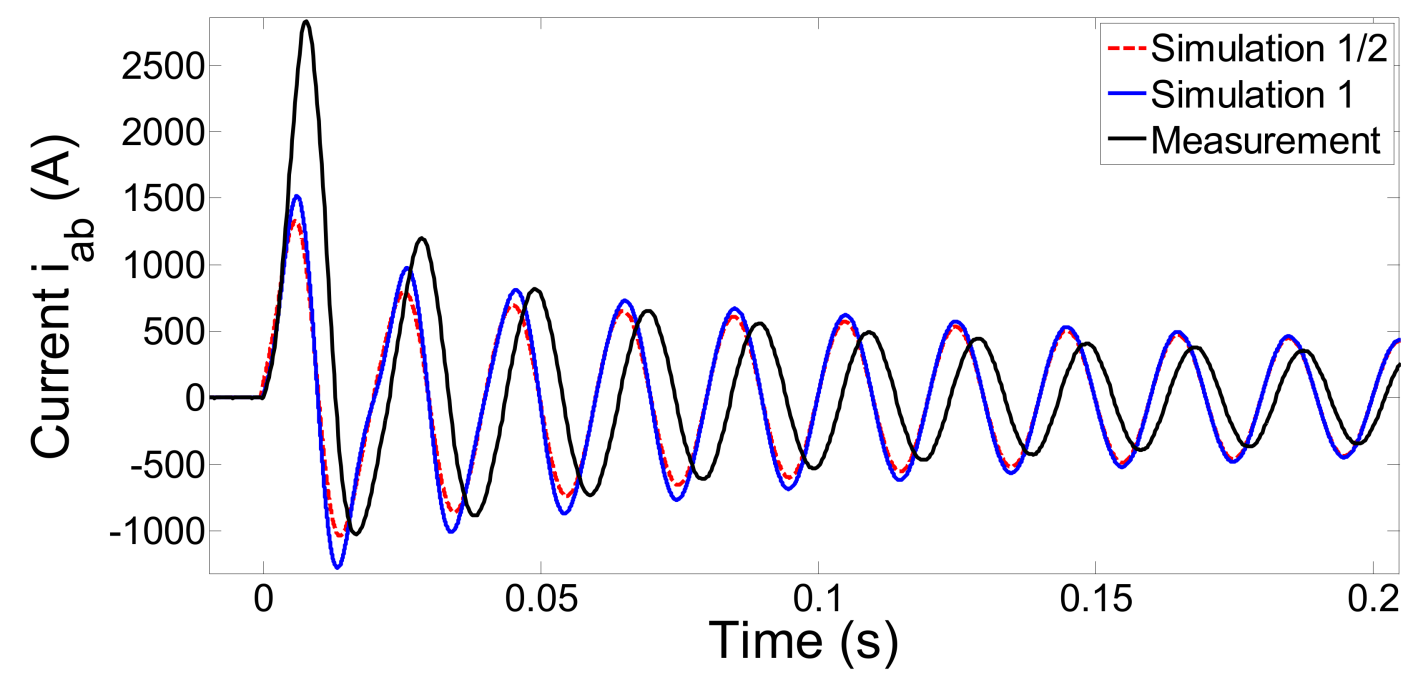

Figure 6. Comparison of simulation and measurement for the short-circuit stator current. 


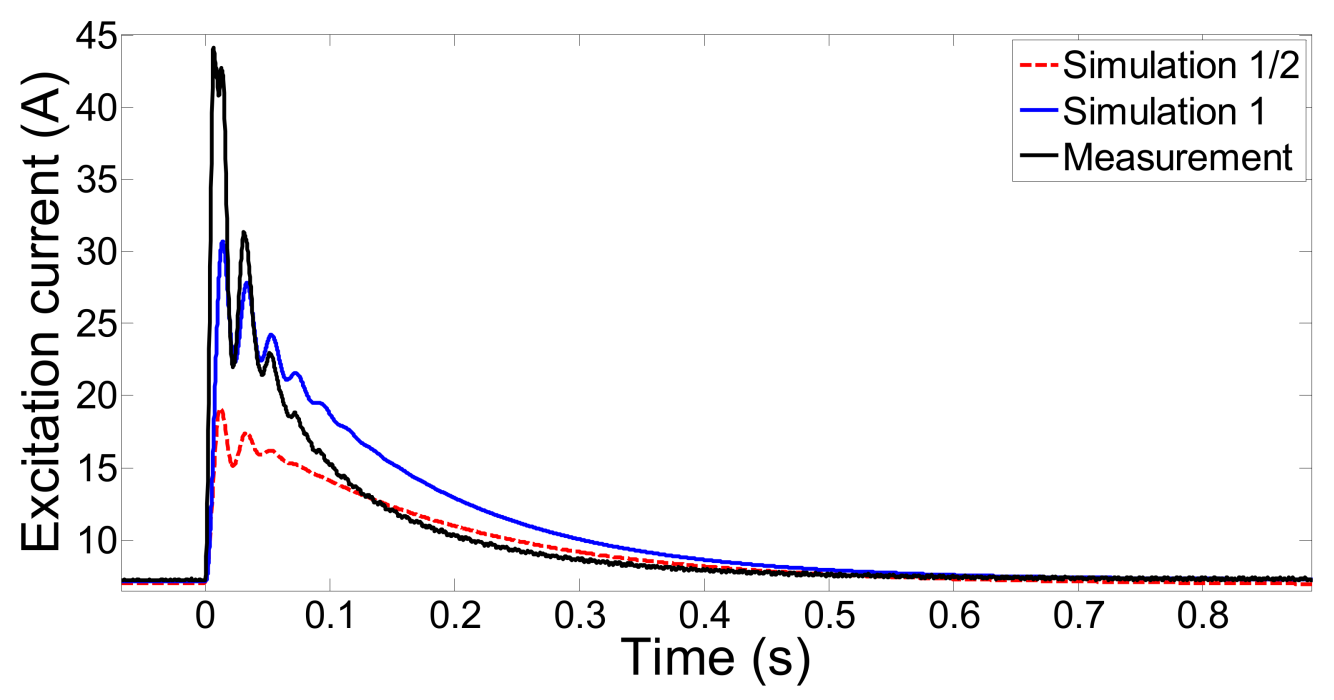

Figure 7. Comparison of simulation and measurement for the short-circuit excitation current.

Figure 8 depicts the simulation of the excitation current $i_{\mathrm{f}}$ during the no-load voltage recovery test (from short-circuit state to no-load conditions) compared to the measurement data. Conclusions are similar to before. Even if the excitation current curve shape modelled by the non-integer order system is correct, it is still less accurate in comparison to the classical integer order one and the real generator dynamics.

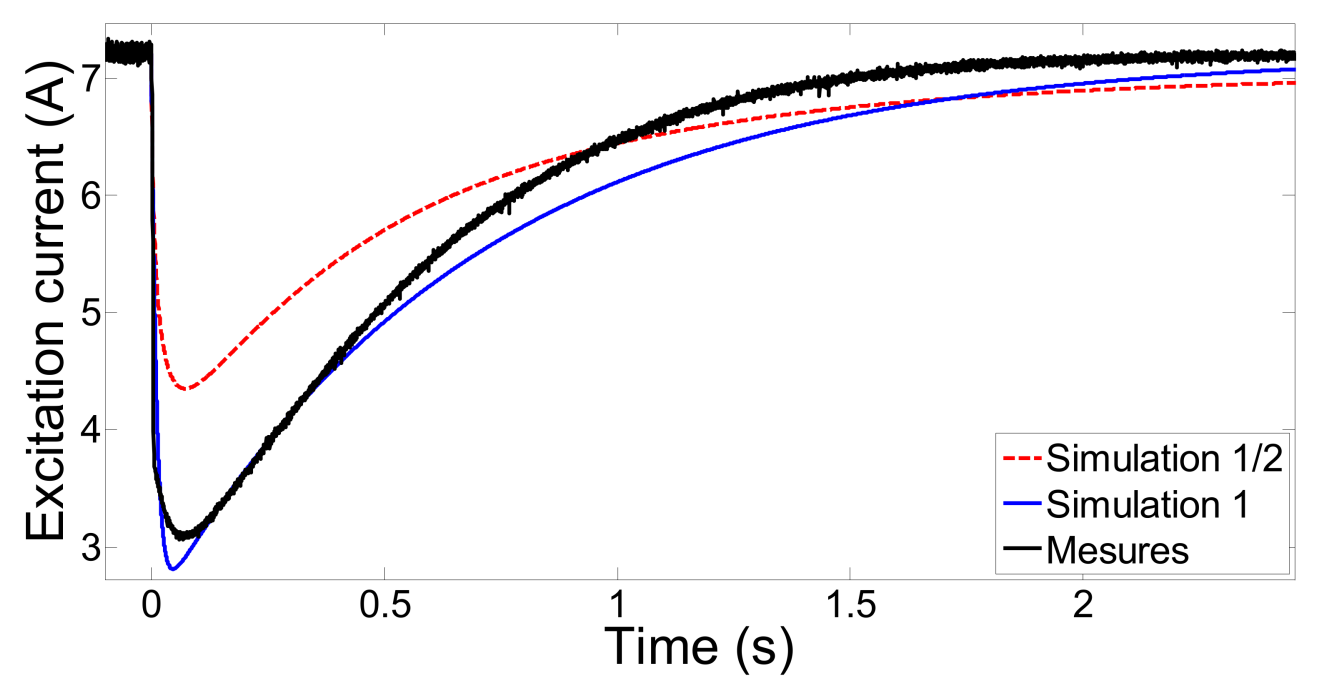

Figure 8. Simulation and measurement of the excitation current during no-load voltage recovery.

\section{Conclusions}

In this work, an analysis of the non-integer order and the classical integer order models of synchronous generator is presented. The comparative study was based on measurement data performed on a $125 \mathrm{kVA}$ synchronous generator. The high performance short-circuit setup consisted of a controlled dynamometer and a power analyser.

Non-integer order derivatives are mathematical tools that have risen in popularity over the last few decades. Non-integer order models are more closely related to the physical aspects of the modelled devices, which on the one hand, improves the modelling accuracy but on the other hand, increases mathematical complexity of the model in the time domain. Indeed, the implicit nature of the models of electric machines leads to the state-space equations comprising a large number of state-space variables [32]. This, in turn, makes the model far more complicated and difficult to 
solve in comparison to the classical, integer order one. Therefore, for the purpose of time domain simulation, the half-order implicit derivatives of the non-integer order model have to be replaced by their explicit equivalents. Thanks to this, the non-integer order derivatives can be easily approximated by, for example, the Grünwald-Letnikov definition. The presented approach can be successfully used for all future non-integer order models when passing from frequency to time domain. Nevertheless, one has to notice that the non-integer order models are originally more suitable and accurate for frequency behaviour description. In electric machine modelling, phenomena associated with frequency, such as eddy currents or skin effect, which are pronounced for example during generator rotor swaying, should be taken into account. The importance of the non-integer order modelling follows from its capability to describe physical phenomena with the fewest parameters possible, without losing the accuracy of the description. This is due to the mathematical nature of the non-integer order derivative, which can take any value because it is not limited to integer order numbers, as in classical integer order derivatives.

The two studied models were identified with SSFR test data. Besides a restricted accuracy of the parameter identification for the classical integer order model, it presents a satisfactory fit in the time domain simulations. Furthermore, such a model does not present any difficulties in calculations or during the simulation procedure as the integer order derivatives are calculated to only one time step back. One should notice that the numerical history of the non-integer order derivatives should be infinite. However, to achieve some compromise between accuracy and simulation time, the number of past derivative values taken into consideration is always limited. In the described example, this value was set to 2500 samples, which caused some slight supplementary error, estimated as $\varepsilon=1.13 \%$ for each calculated derivative.

The main feature of the presented non-integer order modelling method is its structure, based on a physical half-order model of a synchronous machine holding a condensed frequency form for power system stability and network energy quality studies. Having such a frequency model, one can obtain the time response using the procedure proposed and validated in this paper. The main advantage of the proposed model is its accuracy in the frequency domain. The presented results in the time domain only show the capability of the model to be adopted in the time domain. The model accuracy in the time domain would have to be investigated with highly non-sinusoidal time waveforms, such as a non-linear load applied to a synchronous generator, where the model accuracy for higher harmonics could yield better results. This will be investigated in future work.

Author Contributions: Conceptualization, S.R. and F.K.; methodology, S.R. and M.M.; software, S.R.; validation, S.R., M.M., F.K. and Ł.S.; formal analysis, S.R. and Ł.S.; investigation, S.R.; resources, S.R. and M.M.; data curation, S.R.; writing—original draft preparation, S.R.; writing—review and editing, S.R and Ł.S.; visualization, S.R.; supervision, S.R. and F.K.; project administration, S.R. and M.M. All authors have read and agreed to the published version of the manuscript.

Funding: This research received no external funding.

Conflicts of Interest: The authors declare no conflict of interest.

\section{Appendix A}

Synchronous generator Elmor GCh114o/4 rated data:

- Frequency: $f_{n}=50 \mathrm{~Hz}$

- Voltage: $U_{n}=400 \mathrm{~V}$

- Power: $S_{n}=125 \mathrm{kVA}$

Per-unit values:

- Frequency: $f_{b}=f_{n}=50 \mathrm{~Hz}$

- Pulsation: $\omega_{b}=2 \times \pi \times f_{b}=314.1593 \mathrm{rad} / \mathrm{s}$

- Impedance: $Z_{b}=U_{n}{ }^{2} / S_{n}=1.28 \Omega$

- Inductance: $L_{b}=Z_{b} / \omega_{b}=0.0041 \mathrm{H}$ 


\section{References}

1. Oustaloup, A.; Moreau, X.; Nouillant, M. The crone suspension. Control Eng. Pract. 1996, 4, 1101-1108. [CrossRef]

2. Kuhn, E.; Forgez, C.; Friedrich, G. Modeling diffusive phenomena using non integer derivatives application NiMH batteries. EPJ Appl. Phys. 2004, 25, 183-190. [CrossRef]

3. Iftikhar, M.U.; Riu, D.; Druart, F.; Rosini, S.; Bultel, Y.; Retière, N. Dynamic modeling of proton exchange membrane fuel cell using non-integer derivatives. J. Power Sources 2006, 160, 1170-1182. [CrossRef]

4. Martynyuk, V.; Ortigueira, M.D. Fractional model of an electrochemical capacitor. Signal Process. 2015, 107, 355-360. [CrossRef]

5. Lewandowski, M.; Orzyłowski, M. Fractional-order models: The case study of the supercapacitor capacitance measurement. Bull. Pol. Acad. Sci. Tech. Sci. 2017, 65, 449-457. [CrossRef]

6. Skruch, P.; Mitkowski, W. Fractional-Order Models of the Ultracapacitors. In Lecture Notes in Electrical Engineering; Springer Science and Business Media LLC: Berlin, Germany, 2013; Volume 257, pp. 281-293.

7. Jalloul, A.; Trigeassou, J.-C.; Jelassi, K.; Melchior, P. Fractional order modeling of rotor skin effect in induction machines. Nonlinear Dyn. 2013, 73, 801-813. [CrossRef]

8. Riu, D.; Retiere, N.; Ivanes, M. Induced currents modeling by half-order systems application to hydro- and turbo-alternators. IEEE Trans. Energy Convers. 2003, 18, 94-99. [CrossRef]

9. Racewicz, S. Identification and non-integer order modelling of synchronous machines operating as generator. Acta Energ. 2012, 3, 75-84.

10. Kamath, A.; Gandhi, J.; Bohra, A.; Goel, A.V.; Patil, D.; Kulkarni, O.; Chandle, J. Modeling of transformer characteristics using fractional order transfer functions. In Proceedings of the 2009 IEEE International Conference on Control and Automation, Christchurch, New Zealand, 9-11 December 2009; pp. 2123-2128.

11. Oustaloup, A.; Cois, O.; Le Lay, L. Représentation et Identification par Modèle Non Entire; Lavoisier: Paris, France, 2005.

12. Mitkowski, W.; Skruch, P. Fractional-order models of the supercapacitors in the form of RC ladder networks. Bull. Pol. Acad. Sci. Tech. Sci. 2013, 61, 581-587. [CrossRef]

13. Tessarolo, A.; Bassi, C.; Giulivo, D. Time-Stepping Finite-Element Analysis of a 14-MVA Salient-Pole Shipboard Alternator for Different Damper Winding Design Solutions. IEEE Trans. Ind. Electron. 2011, 59, 2524-2535. [CrossRef]

14. Racewicz, S. Modelisation d'Ordre Non Entier des Machines Synchrones; Éditions Universitaires Européennes: Sarrebruck, Germany, 2011.

15. Oustaloup, A.; Cois, O.; Lanusse, P.; Melchior, P.; Moreau, X.; Sabatier, J. The Crone Aproach: Theoretical Developments and Major Applications. IFAC Proc. Vol. 2006, 39, 324-354. [CrossRef]

16. Podlubny, I. Fractional Differential Equations. An Introduction to Fractional Derivatives, Fractional Differential Equations, to Methods of their Solution and Some of Their Applications; Academic Press: Cambridge, MA, USA, 1999.

17. Brzeziński, D.W.; Ostalczyk, P. About accuracy increase of fractional order derivative and integral computations by applying the Grünwald-Letnikov formula. Commun. Nonlinear Sci. Numer. Simul. 2016, 40, 151-162. [CrossRef]

18. Scherer, R.; Kalla, S.L.; Tang, Y.; Huang, J. The Grünwald-Letnikov method for fractional differential equations. Comput. Math. Appl. 2011, 62, 902-917. [CrossRef]

19. Krause, P.C.; Wasynczuk, O.; Sudhoff, S.D. Analysis of Electric Machinery and Drive Systems; Wiley-IEEE Press: Hoboken, NJ, USA, 2013.

20. Wamkeue, R.; Kandil, N.; Berrada, M. Equivalent circuit based current-controlled state model of synchronous machine. In Proceedings of the IPEMC 2004: 4th International Power Electronics and Motion Control Conference, Xi'an, China, 14-16 August 2004; Volume 3, pp. 1483-1489.

21. Radjeai, H.; Abdessemed, R.; Tnani, S.; Mouni, E. A method to improve the synchronous machines equivalent circuits. In Proceedings of the EUROCON 2007-The International Conference on Computer as a Tool, Warsaw, Poland, 9-12 September 2007; pp. 2367-2372. 
22. Hiramatsu, D.; Uemura, Y.; Okumoto, J.; Uemoto, S.; Imai, T.; Kakiuchi, M.; Nagakura, K.; Otaka, T.; Fujita, M.; Nagasaka, K. A study on quadrature equivalent circuit model in large synchronous machine. In Proceedings of the IEEE Power and Energy Society 2008 General Meeting: Conversion and Delivery of Electrical Energy in the 21st Century, Pittsburgh, PA, USA, 20-24 July 2008.

23. Ma, Y.; Zhou, L.; Wang, J.; Cui, Y.; Cai, W.; Zhao, J. Accurate equivalent circuit modeling and identification of solid rotor synchronous machine. In Proceedings of the 1st IEEE Student Conference on Electric Machines and Systems, Huzhou, China, 14-16 December 2018.

24. Racewicz, S.; Riu, D.M.; Retiere, N.M.; Chrzan, P.J. Half-order modelling of ferromagnetic sheet. In Proceedings of the 2011 IEEE International Symposium on Industrial Electronics, Gdansk, Poland, 27-30 June 2011; pp. 607-612.

25. Racewicz, S.; Riu, D.M.; Retière, N.M.; Chrzan, P.J. Half-order modelling of turboalternators-An adapted method of parameter identification. In Proceedings of the International Conference on Electrical Machines, Chania, Crete Island, Greece, 2-5 September 2006; pp. 1-6.

26. Racewicz, S.; Riu, D.; Retière, N.; Chzran, P. Non linear half-order modeling of synchronous machine. In Proceedings of the 2009 IEEE International Electric Machines and Drives Conference, Miami, FL, USA, 3-6 May 2009; pp. 778-783.

27. Racewicz, S.; Chrzan, P.J.; Riu, D.M.; Retiere, N.M. Time domain simulations of synchronous generator modelled by half-order system. In Proceedings of the IECON 2012-38th Annual Conference on IEEE Industrial Electronics Society, Montreal, QC, Canada, 25-28 October 2012; pp. 2074-2079.

28. IEEE Guide. Test Procedures for Synchronous Machines Part I-Acceptance and Performance Testing Part II-Test Procedures and Parameter Determination for Dynamic Analysis; IEEE Std: Piscataway, NJ, USA, 2010; Volume 115.

29. Almeida, R.; Tavares, D.; Torres, D.F. The Variable-Order Fractional Calculus of Variations; Springer International Publishing: Cham, Switzerland, 2019.

30. Li, Z.; Liu, L.; Dehghan, S.; Chen, Y.; Xue, D. A review and evaluation of numerical tools for fractional calculus and fractional order controls. Int. J. Control. 2016, 90, 1165-1181. [CrossRef]

31. Matusiak, M.; Ostalczyk, P. Problems in solving fractional differential equations in a microcontroller implementation of an FOPID controller. Arch. Electr. Eng. 2019, 68, 565-577.

32. Mansouri, R.; Bettayeb, M.; Djennoune, S. Approximation of high order integer systems by fractional order reduced-parameters models. Math. Comput. Model. 2010, 51, 53-62. [CrossRef] 